AS-TEXONO/06-06

23 September 2018

\title{
Research program towards observation of neutrino-nucleus coherent scattering
}

\author{
H T Wong ${ }^{1, *}, \mathbf{H} \mathbf{B} \mathbf{L i}^{1}, \mathbf{S} \mathbf{K} \mathbf{L i n}^{1}, \mathbf{S} \mathbf{T} \mathbf{L i n}^{1}, \mathbf{D} \mathbf{H e}^{2}, \mathbf{J ~ L i}^{2}, \mathbf{X} \mathbf{L i}^{2}, \mathbf{Q}$ \\ $\mathrm{Yue}^{2}, \mathrm{Z}$ Y Zhou ${ }^{3}$ and S K Kim ${ }^{4}$ \\ ${ }^{1}$ Institute of Physics, Academia Sinica, Taipei 11529, Taiwan. \\ 2 Department of Engineering Physics, Tsing Hua University, Beijing 100084, China. \\ 3 Department of Nuclear Physics, Institute of Atomic Energy, Beijing 102413, China. \\ ${ }^{4}$ Department of Physics, Seoul National University, Seoul 151-742, Korea. \\ E-mail: htwong@phys.sinica.edu.tw (*Corresponding Author)
}

\begin{abstract}
The article describes the research program towards an experiment to observe coherent scattering between neutrinos and the nucleus at the power reactor. The motivations of studying this process are surveyed. In particular, a threshold of 100-200 eV has been achieved with an ultra-low-energy germanium detector prototype. This detection capability at low energy can also be adapted for searches of Cold Dark Matter in the low-mass region as well as to enhance the sensitivities in the study of neutrino magnetic moments.
\end{abstract}

Neutrino coherent scattering with the nucleus [1]

$$
\nu+\mathrm{N} \rightarrow \nu+\mathrm{N}
$$

is a fundamental neutrino interaction which has never been experimentally observed. The Standard Model cross section for this process is given by:

$$
\begin{gathered}
\left(\frac{\mathrm{d} \sigma}{\mathrm{dT}}\right)_{\mathrm{SM}}^{\mathrm{coh}}=\frac{\mathrm{G}_{\mathrm{F}}^{2}}{4 \pi} \mathrm{m}_{\mathrm{N}}\left[\mathrm{Z}\left(1-4 \sin ^{2} \theta_{\mathrm{W}}\right)-\mathrm{N}\right]^{2}\left[1-\frac{\mathrm{m}_{\mathrm{N}} \mathrm{T}_{\mathrm{N}}}{2 \mathrm{E}_{\nu}^{2}}\right] \\
\sigma_{\text {tot }}=\frac{\mathrm{G}_{\mathrm{F}}^{2} \mathrm{E}_{\nu}^{2}}{4 \pi}\left[\mathrm{Z}\left(1-4 \sin ^{2} \theta_{\mathrm{W}}\right)-\mathrm{N}\right]^{2}
\end{gathered}
$$

where $\mathrm{m}_{\mathrm{N}}, \mathrm{N}$ and $\mathrm{Z}$ are the mass, neutron number and atomic number of the nuclei, respectively, $\mathrm{E}_{\nu}$ is the incident neutrino energy and $\mathrm{T}_{\mathrm{N}}$ is the measure-able recoil energy of the nucleus. This formula is applicable for $\mathrm{E}_{\nu}<50 \mathrm{MeV}$ where the momentum transfer $\left(\mathrm{Q}^{2}\right)$ is small such that $\mathrm{Q}^{2} \mathrm{R}^{2}<1$, where $\mathrm{R}$ is the nuclear size. Although the cross-section is relatively large due to the $\sim \mathrm{N}^{2}$ enhancement by coherence, the small kinetic energy from nuclear recoils poses severe experimental challenges both to the detector sensitivity and to background control. Various detector techniques have been considered 2] to meet these challenges.

Measurement of the coherent scattering cross-section would provide a sensitive test to the Standard Model[3], probing the weak nuclear charge and radiative corrections due to possible new physics above the weak scale. The coherent interaction plays important role in astrophysical processes where the neutrino-electron scatterings are suppressed due to Fermi gas degeneracy. 
It is significant to the neutrino dynamics and energy transport in supernovae and neutron stars 4. Being a new detection channel for neutrinos, it may provide new approaches to study other aspects of neutrino physics, such as that for supernova neutrinos [5]. Coherent scattering with the nuclei is also the detection mechanism adopted in the direct Dark Matter searches [ 6 , such that its observations and measurements with the known particle neutrino is an important milestone. Furthermore, neutrino coherent scattering may be a promising avenue towards a compact and relatively transportable neutrino detector, an application of which can be for the real-time monitoring on the operation of nuclear reactors [7].

Nuclear power reactors provide powerful and controllable source of electron anti-neutrinos, and can serve as optimal tool for the studies of neutrino-nucleus scatterings. A research program on low energy neutrino physics $[\underline{8}$ is intensely pursued by the TEXONO Collaboration at the Kuo-Sheng (KS) Nuclear Power Station in Taiwan. The expected observable spectra due to $\overline{\nu_{\mathrm{e}}}-\mathrm{e}$ and $\overline{\overline{\mathrm{e}}_{\mathrm{e}}}-\mathrm{N}$ scatterings with Standard Model $(\mathrm{SM})$ and magnetic moment (MM) interactions at KS are displayed in Figure 1. The maximum nuclear recoil energy $\mathrm{T}_{\max }$ in $\overline{\mathrm{\nu}_{\mathrm{e}}}-\mathrm{N}$ coherent scatterings is given by:

$$
\mathrm{T}_{\max }=\frac{2 \mathrm{E}_{\nu}^{2}}{\mathrm{M}_{\mathrm{N}}+2 \mathrm{E}_{\nu}}
$$

which corresponds to $\mathrm{T}_{\max }=1.9 \mathrm{keV}$ in the case of Ge target $(\mathrm{A}=72.6)$ exposed to the typical reactor neutrino spectra.

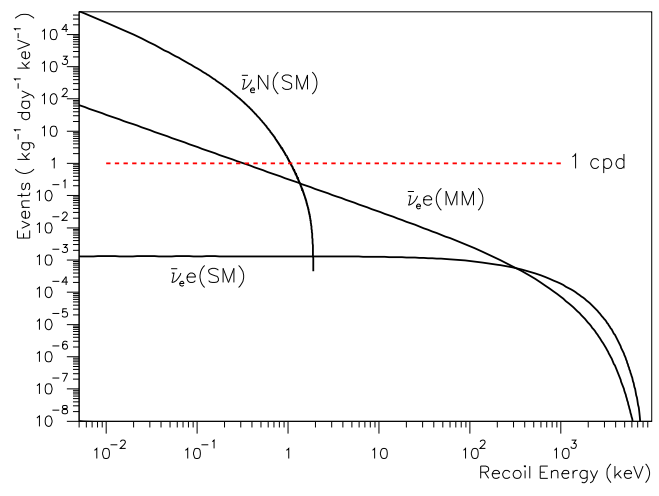

Figure 1. The differential cross-section of the various neutrino interaction channels, at KS-Lab with Ge as the target isotope. The background level of $1 \mathrm{cpd}$ is also shown.

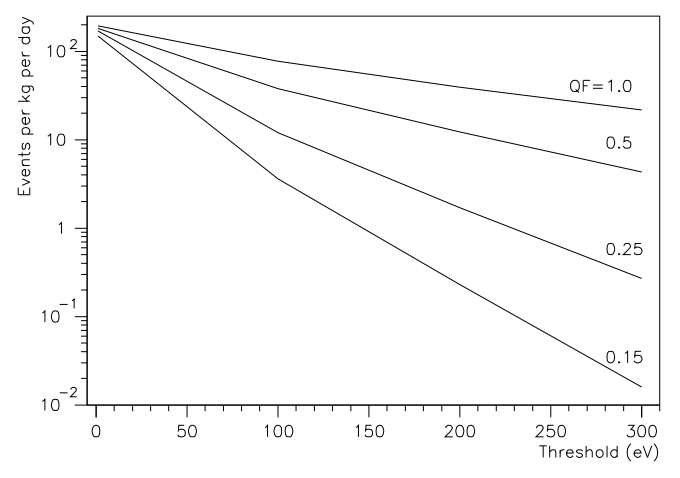

Figure 2. The variations of the neutrino coherent scattering event rates versus threshold at different quenching factors for a $1 \mathrm{~kg}$ ULEGe detector at KS Lab.

High-Purity Germanium(HPGe) detectors have been widely and successfully used in various areas of low energy neutrino physics and cold dark matter searches. These detectors are kilogram-scale in mass, and with a detection threshold ("noise edge") of several keV. A sensitive direct search of neutrino magnetic moments 9 was recently performed with a $1 \mathrm{~kg}$ HPGe detector at KS[10]. A physics threshold of $12 \mathrm{keV}$ and a background level of $\sim 1 \mathrm{day}^{-1} \mathrm{~kg}^{-1} \mathrm{keV}^{-1}$ (cpd) comparable to underground dark matter experiments were achieved. For ionization detectors like germanium, the measure-able energy of nuclear recoil events is only a fraction of their energy deposited due to charge recombination or "quenching" at large $\mathrm{dE} / \mathrm{dx}$. The expected event rates for neutrino-nucleus coherent scattering at different threshold and quenching factors (QF) at KS are depicted in Figure 2. The QF for Ge is typically 0.25 in the several keV region, such that the maximum measure-able energy for nuclear recoil events is only about $500 \mathrm{eV}$.

"Ultra-Low-Energy" Germanium (ULEGe) detectors, developed originally for soft X-rays detection, are candidate technologies to meet these challenges of probing into a previously 
unexplored energy domain[1]. These detectors typically have modular mass of 5-10 grams while detector array of up to $\mathrm{N}=30$ elements have been successfully built. Various prototypes based on this detector technology have been constructed. As illustrations, the measured energy spectrum with a $10 \mathrm{~g}$ ULEGe prototype is depicted in Figure 3. Pulse shape discrimination (PSD) criteria were applied as illustrated in Figure 4 by correlating two output with different electronics amplifications and shaping times. Calibration was achieved by external ${ }^{55} \mathrm{Fe} \mathrm{X}$-ray sources $(5.90$ and $6.49 \mathrm{keV})$ together with X-rays from titanium $(4.51$ and $4.93 \mathrm{keV})$, calcium (4.01 keV), sulphur $(2.46 \mathrm{keV})$ and aluminium $(1.55 \mathrm{keV})$. A random trigger uncorrelated to the detector provided the zero-energy condition. The electronic noise edge can be suppressed by PSD and a threshold of $100 \mathrm{eV}$ was achieved.

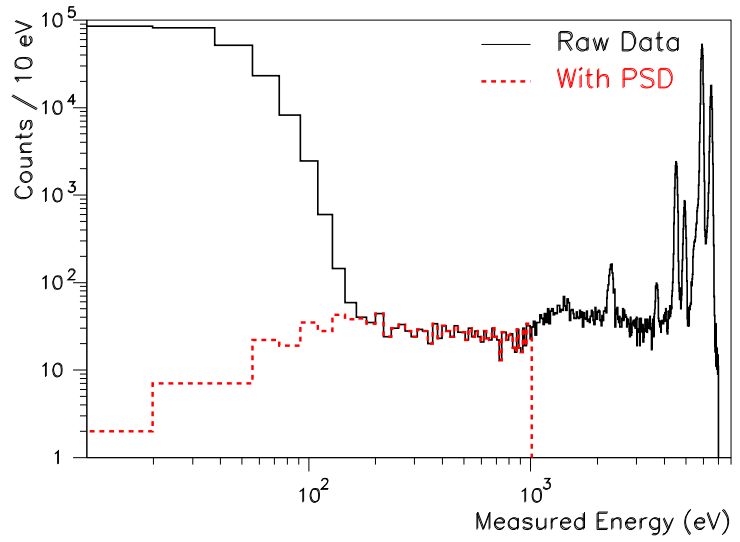

Figure 3. Measured energy spectra with ${ }^{55} \mathrm{Fe}$ source with $\mathrm{X}$-rays from $\mathrm{Ti}$ by the ULEGe prototype. A threshold of $100 \mathrm{eV}$ was achieved, and the electronic noise edge was suppressed by PSD.

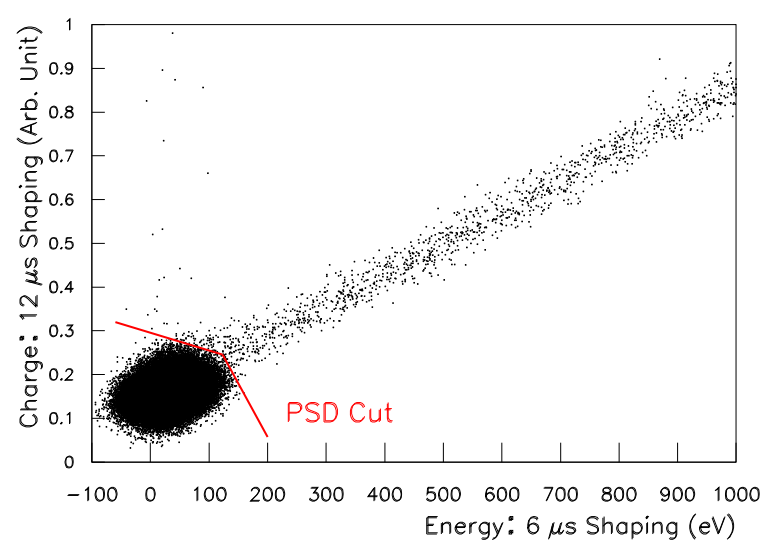

Figure 4. Pulse shape discrimination: correlations of signals with different electronic amplifications and shaping times lead to suppression of the noise edge.

The goal of the $\nu$-N coherent scattering experiment is to develop a ULEGe detector with a total mass of $\sim 1 \mathrm{~kg}$ and a modular threshold as low as $100 \mathrm{eV}$, with a background level below $1 \mathrm{keV}$ in the range of $1 \mathrm{cpd}$. From Figure 2 and at the typical $\mathrm{QF}=0.25$, the event rate for such configurations at KS will be $11 \mathrm{~kg}^{-1}$ day $^{-1}$, implying a signal-to-background ratio of $>22$. A by-product of such an detector would be to further enhance the searches of neutrino magnetic moment at reactors. An improved sensitivity range down to $\sim 10^{-11} \mu_{B}$ can be expected [9]. Such detector can also be used for Cold Dark Matter searches $[\underline{6}$, probing the unexplored low WIMP-mass region, as indicated in Figure 5 .

An R\&D program towards realizations of these experiments is being pursued. A quenching factor measurement for nuclear recoils in Ge with sub-keV ionization energy is being prepared at a neutron beam facility. Background studies are performed at both KS as well as the Yang-Yang Underground Laboratory (Y2L) in South Korea with the different prototypes. The measured spectra above $1 \mathrm{keV}$ on the various configurations are presented in Figure [6] while sub-keV background are under intense studies. The factor-of-ten difference in the mass-normalized background levels between the $1 \mathrm{~kg}$ and $5 \mathrm{~g}$ detectors is due to self-shielding of the detector. Such effects are reproduced by realistic simulation studies on the variations of $\gamma$ and neutron background levels with detector mass, as depicted in Figure 7

Consequently, in the scaling-up to the kilogram mass-range, the individual elements of the ULEGe should be assembled in a compact array in order to maintain the same background level of $\sim 1$ cpd achieved in the $1 \mathrm{~kg}$ HPGe detector. The "segmented" Ge technology with the 


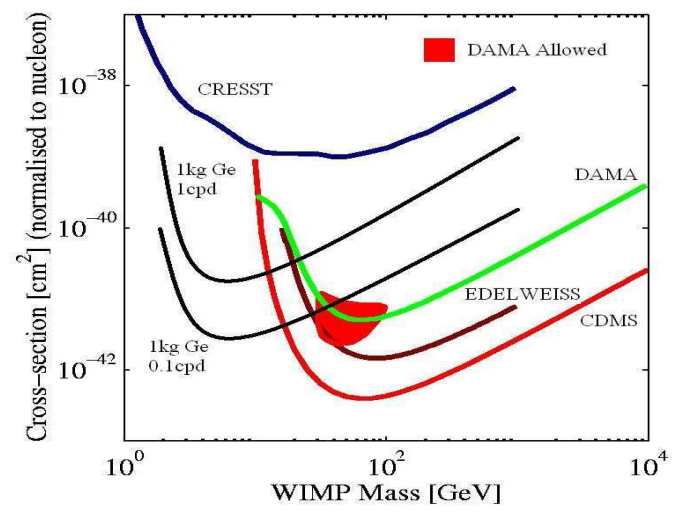

Figure 5. Expected sensitivity region for Cold Dark Matter searches using a ULEGe detector with a total mass of $1 \mathrm{~kg}$.

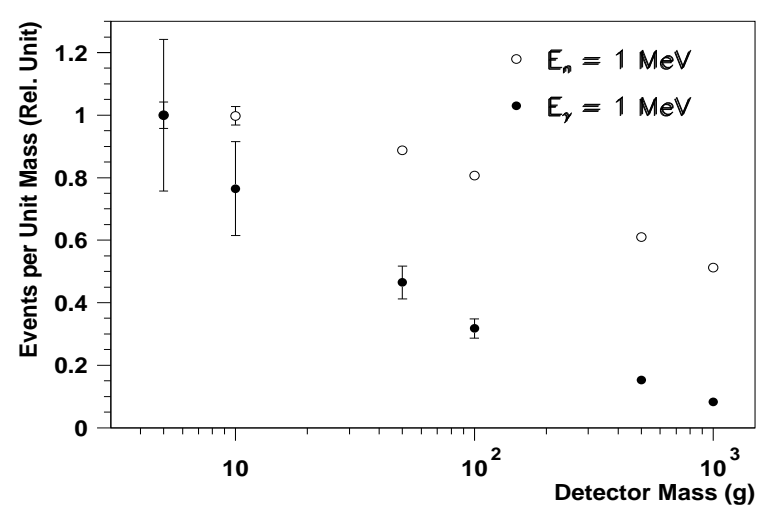

Figure 7. Simulated results on the variations of background per unit mass versus detector mass at the same external $\gamma$ and neutron background level.

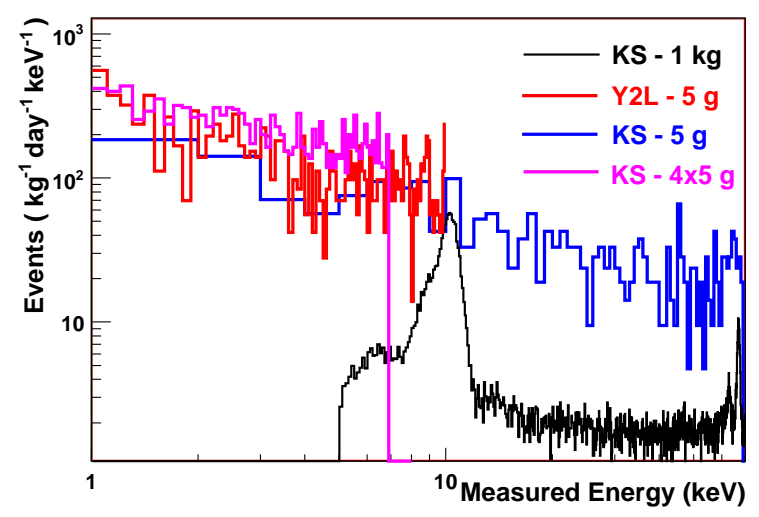

Figure 6. Measured background spectra, normalized by mass, at KS and Y2L with different Ge detectors.

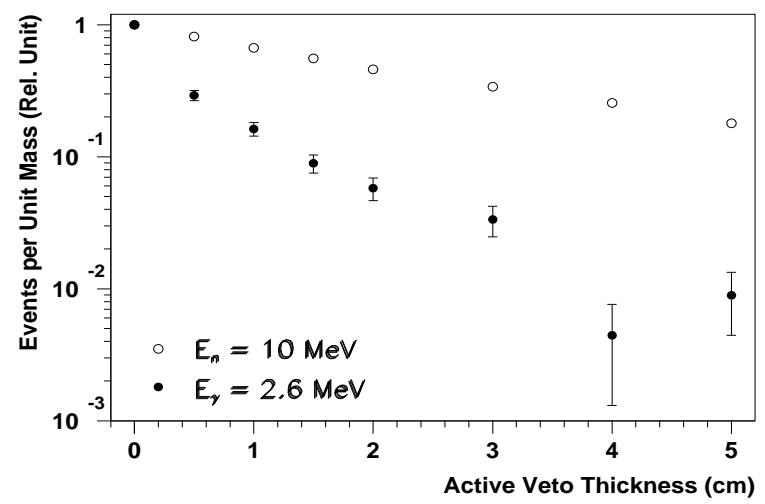

Figure 8. Simulated results on the background suppression for $\gamma$ and neutron sources at the inner ULEGe target due to an enclosing structures of active Ge-vetos.

integrated circuitry approach is an attractive alternative. Configurations can be envisaged to have active veto HPGe layers enclosing hermetically the ULEGe inner target. Simulation results of Figure 8 show that a Ge-veto thickness of $3 \mathrm{~cm}$ can further suppress the background at the inner target by about a factor of 30 and 3 for typical $\gamma$ and neutron background, respectively. A prototype segmented ULEGe detector with a veto ring and dual-readout channels from both the signal and high-voltage electrodes is being constructed.

\section{References}

[1] Freedman D S 1974 Phys. Rev. D 9, 1389; Gaponov Y V and Tikhonov V N 1977 Sov. J. Nucl. Phys. 26, 31; Sehgal L H and Wanninger M 1986 Phys. Lett. B 171, 107.

[2] Drukier A and Stodolsky L 1984 Phys. Rev. D 30, 2295; Canbrea B, Krauss L M and Wilczek F 1985 Phys. Rev. Lett. 55, 25; Barbeau P et al. 2003 IEEE Tran. Nucl. Sci. 50, 1285; Hagmann C and Bernstein A, 2004 IEEE Tran. Nucl. Sci. 51, 2151.

[3] Krauss L M 1991 Phys. Lett. B 269, 407; Barranco J, Miranda O G and Rashba T I 2005 J. High Energy Phys. 12, 021; Papavassiliou J, Bernabéu J and Passera M 2005 Preprint hep-ph/0512029 Scholberg K 2006 Phys. Rev. D 73, 033005. 
[4] Wilson J R 1974 Phys. Rev. Lett. 32, 849; Freedman D Z, Schramm D N and Tubbs D L 1977 Annu. Rev. Nucl. Sci. 27, 167.

[5] Aune S et al. 2005 Preprint hep-ex/0503031 Scholberg K, these Proceedings.

[6] Akerid D 2006 these Proceedings; Smith N 2006 these Proceedings;

[7] Cribier M 2006 these Proceedings.

[8] Wong H T 2004 Mod. Phys. Lett. A 19, 1207.

[9] Wong H T and Li H B 2005 Mod. Phys. Lett. A 20, 1103.

[10] Li H B et al. 2003 Phys. Rev. Lett. 90, 131802; Xin B et al. 2005 Phys. Rev. D 72, 012006 ; Wong H T 2006 Preprint hep-ex/0605006

[11] Wong H T et al. 2006 J. Phys. Conf. Ser. 39, 266. 